\title{
CFD Validation for High-Lift Devices: Two-Element Airfoil*
}

\author{
By Zhong LeI ${ }^{1)}$ Mitsuhiro Murayama, ${ }^{1)}$ Keizo TaKenaKa ${ }^{2)}$ and Kazuomi Yamamoto ${ }^{1)}$ \\ ${ }^{1)}$ Aviation Program Group, Japan Aerospace Exploration Agency, Tokyo, Japan \\ ${ }^{2)}$ Nagoya Aerospace Systems, Mitsubishi Heavy Industries, Ltd., Nagoya, Japan
}

(Received April 14th, 2005)

\begin{abstract}
The objectives of this study are to assess the accuracy of CFD codes, investigate the effect of turbulence models as applied to the flow around high-lift devices, and increase the knowledge for computing this kind of flow. CFD validation is conducted for a two-element airfoil and compared with a wind tunnel test to predict the aerodynamic forces, including the maximum lift and the stall angle. Sensitivity to grid density and influence of the grid extent are investigated. Four RANS codes with the same turbulence model are tested and computational results are compared with each other. Three commonly used turbulence models implemented in a CFD code are applied to investigate the effects of turbulence models for this kind of high-lift flow.
\end{abstract}

Key Words: High-lift, Two-dimensional, RANS, Turbulence Model

\section{Introduction}

In an aircraft design, the development of effective highlift devices is one of the most important requirements for successful design. High-lift devices commonly used are leading-edge slats and trailing-edge flaps which increase the wing area and effective camber to generate sufficient low-speed performance in take-off and landing situations. The high-lift flow around a multi-element airfoil is complicated by a number of important flow phenomena such as separation, transition, wake/boundary layer confluence, shock waves, etc. With the development of computer and numerical algorithms, computational fluid dynamics (CFD) has been widely accepted as an important factor in reducing cost in the design process and shortening time to market.

CFD research for high-lift flows was reviewed recently by Rumsey and Ying. ${ }^{1)}$ Accurate predictions of aerodynamic characteristics, especially stall behavior, are required for designing a high-lift system. Numerical simulations of highlift flows by solving Reynolds-averaged Navier-Stokes (RANS) equations are now often used in the analysis and design of high-lift devices. Due to the complexity of geometries and high computation cost, less progress has been made in applying CFD simulations to three-dimensional (3D) designs. Although high-lift flows are inherently 3D, many aspects of such flows may be understood by studying simplified two-dimensional (2D) problems. Simplified 2D analysis may be helpful to understand such flow physics and it can be conducted much faster than 3D analysis. Obviously, the performance of a 2D high-lift device differs from that in 3D. Beginning with a good 2D design will certainly shorten the $3 \mathrm{D}$ analysis and design process. However, there are many problems associated with CFD that influence the accu-

(C) 2006 The Japan Society for Aeronautical and Space Sciences

*Presented at 2004 KSAS-JSASS Joint Symposium on Aerospace Engineering, Nov. 18-19, Seoul, Korea. racy of prediction. Even in two dimensions it is still difficult to predict this kind of flow.

The objectives of this study are to assess the accuracy of CFD codes, investigate the effect of turbulence models as applied to the high-lift flow, and increase the knowledge for computing this kind of flow. This paper discusses CFD prediction for high-lift flow around a two-element airfoil. Computations of a three-element airfoil are given separately in a companion paper. ${ }^{2)}$

Because of the complex geometry and flow phenomena, there are many dependence factors that influence the prediction accuracy in the computation of high-lift flow. Although prediction of turbulence transition has a relatively large effect on computational results, we do not intend to validate transition models in this paper. In this study, transition onsets are specified according to experimental observation. For discussion about the effect of transition on the computational results, see Kusunose ${ }^{3)}$ and Krumbein. ${ }^{4)}$ We focus only on the discussion of three topics that are very important in predicting the accuracy of high-lift flow.

1. Grid dependency: To investigate discretization error in CFD simulation, a successive grid refinement study is carried out.

2. Solver comparison: RANS equations were solved by four different codes associated with the same turbulence model (i.e., Spalart-Allmaras model). Four solvers employed different discrete schemes and were performed on different mesh types.

3. Effects of turbulence models: To minimize other differences such as grid refinement and numerical discretization, comparisons were conducted using a solver with three turbulence models incorporated.

\section{Description of Test Case: Two-Element Airfoil}

The test case is chosen as a two-element high-lift configuration based on the NLR7301 airfoil, as shown in Fig. 1, 
Table 1. Summary of CFD solvers.

\begin{tabular}{llllll}
\hline Code name & Discretization & Mesh type & Mesh size & Turbulence model & Transition \\
\hline \multirow{2}{*}{ UPACS } & $\begin{array}{l}\text { 2nd-order in space } \\
\text { MFGS for time } \\
\text { integration }\end{array}$ & $\begin{array}{l}\text { multi-block } \\
\text { structured }\end{array}$ & 414,000 & SA & fixed \\
& $\begin{array}{l}\text { 2nd-order in space } \\
\text { LU-SGS for time } \\
\text { integration }\end{array}$ & unstructured & 250,000 & SA & fixed \\
\hline TAS & $\begin{array}{l}\text { 2nd-order in space } \\
\text { LU-ADI for time } \\
\text { integration }\end{array}$ & $\begin{array}{l}\text { structured } \\
\text { overset }\end{array}$ & 216,000 & SA & $\begin{array}{l}\text { fixed on main } \\
\text { element } \\
\text { free on flap }\end{array}$ \\
\hline \multirow{2}{*}{ ADCS } & $\begin{array}{l}\text { 2nd-order in space } \\
\text { LU-ADI for time } \\
\text { integration }\end{array}$ & $\begin{array}{l}\text { multi-block } \\
\text { structured }\end{array}$ & 124,000 & SA, $k-\varepsilon$, SST & fixed \\
\hline
\end{tabular}

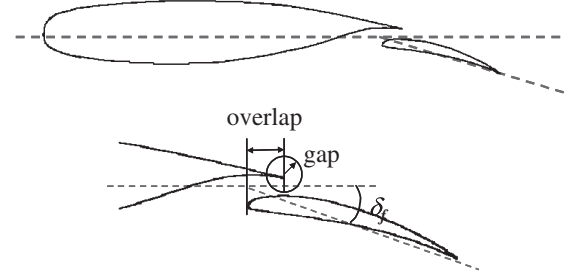

Fig. 1. Configuration of the two-element airfoil NLR7301.

which was tested in the NLR $3 \mathrm{~m} \times 2 \mathrm{~m}$ low-speed wind tunnel in $1970 \mathrm{~s}^{5)}$ It is one of the most computed multi-element airfoils. This configuration was designed with a moderate flap angle of $20^{\circ}$ so that no flow separation would occur on the flap, except a small laminar separation bubble on the wing nose. Wind tunnel tests were conducted at a freestream Mach number of 0.185 and Reynolds number of $2.51 \times 10^{6}$ based on the chord of the basic airfoil. Measurements were performed for two configurations with different values of gap width, $1.3 \%$ and $2.6 \%$ chord, between the main element and the flap. For both configurations, the overlap of the main element and flap was the same, 5.3\% chord. It is representative of a typical setting in take-off situation. Pressure distribution was measured by a number of static pressure holes on the model surface. Based on the assumption that the log-law of the wall holds in a flow region near the wall, a limited number of skin friction values were obtained with the razor-blade technique. The lift and the moment were then obtained from an integration of the pressure measured on the surface. The drag was derived from wake traverses at one chord distance downstream of the trailing edge. The boundary layer profiles were obtained using a boundary layer probe. Transition on the model surface was observed using the sublimation technique. All the measurement data were documented and can be obtained for use in validation of numerical computation. ${ }^{5,6)}$ The $1.3 \%$ gap case was selected.

\section{Flow Solver and Turbulence Model}

Four codes are used to solve the compressible RANS equations with eddy-viscosity turbulence models on differ- ent type of meshes. All codes globally have 2nd-order accuracy in spatial discretization. The main features of solvers and corresponding meshes are summarized in Table 1 . In this study, three turbulence models, Spalart-Allmaras one equation model (SA), Menter's shear stress transport twoequation model (SST), and Abid $k-\varepsilon$ two-equation model $(k-\varepsilon)$ are used to simulate turbulent flows.

Two kinds of in-house codes (UPACS and ADCS) are used on multi-block structured meshes. The Unified Platform for Aerospace Computational Simulation (UPACS $)^{7,8)}$ is a standard CFD code for aeronautic flows in the Institute of Space Technology and Aeronautics of Japan Aerospace Exploration Agency (JAXA). The UPACS solver is based on a cell-centered finite volume method. The AeroDynamic Computational $\underline{\text { System }}$ (ADCS) ${ }^{9)}$ is also developed in JAXA. It is based on a finite difference method. As the unstructured mesh generator and flow solver, The Tohoku university Aerodynamic $\underline{\text { Simulation }}$ (TAS) code ${ }^{10)}$ is used in this study. RANS equations are solved on the unstructured mesh using a cell-vertex finite volume method. MHI2D ${ }^{11)}$ is a two-dimensional CFD code developed by Mitsubishi Heavy Industries. It solves RANS equations using the finite difference method on structured mesh with overset technique.

The Spalart-Allmaras one-equation model ${ }^{12)}$ is calibrated to a wide range of experiment data, and is likely to be applicable to a fair range of thin shear layer flows. A variation of the model, SARC, ${ }^{13)}$ which suppresses unphysical high eddy viscosity using rotation correction in the regions of high vorticity, is also used in ADCS. For two-dimensional flows with small separation, the difference between SA and SARC models are generally small. Test computation shows that the drag difference between SA and SARC models is less than 0.0002. The Menter's SST model ${ }^{14)}$ combined the $k-\varepsilon$ and $k-\omega$ models in a way that allowed them to be used in regions where they achieve the best advantage. The model uses the $k-\omega$ model near the wall, and switches to the $k-\varepsilon$ model away from the wall. Furthermore, the model limits the shear stress according to experimental observation. Studies showed that this limitation significantly improved results in separated flows. Among eddy-viscosity models, this model performs fairly well in many applications. The 
Table 2. Transition locations on airfoil surface.

\begin{tabular}{|c|c|c|c|c|c|}
\hline \multirow{2}{*}{ AoA } & & \multicolumn{2}{|c|}{ Main element } & \multicolumn{2}{|c|}{ Flap } \\
\hline & & upper & lower & upper & lower \\
\hline \multirow{2}{*}{6.0} & experiment & $0.027 \sim 0.040$ & $0.635 \sim 0.675$ & $1.063 \sim 1.081$ & laminar \\
\hline & CFD & $x \approx 0.035$ & $x \approx 0.652$ & $x \approx 1.060$ & laminar \\
\hline \multirow{2}{*}{10.1} & experiment & $0.025 \sim 0.037$ & $0.680 \sim 0.710$ & $1.054 \sim 1.072$ & laminar \\
\hline & CFD & $x \approx 0.032$ & $x \approx 0.692$ & $x \approx 1.054$ & laminar \\
\hline \multirow{2}{*}{13.1} & experiment & $0.024 \sim 0.035$ & $0.685 \sim 0.715$ & $1.047 \sim 1.064$ & laminar \\
\hline & CFD & $x \approx 0.029$ & $x \approx 0.705$ & $x \approx 1.048$ & laminar \\
\hline
\end{tabular}

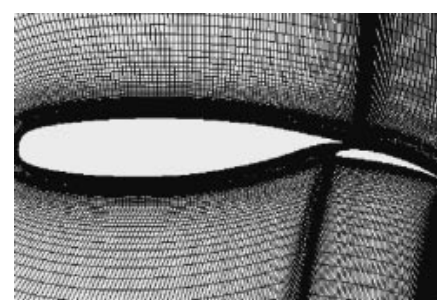

(a) Structured mesh of UPACS code

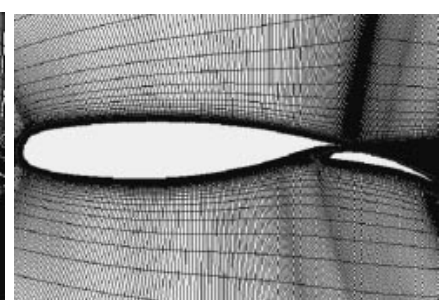

(b) Structured mesh of ADCS code

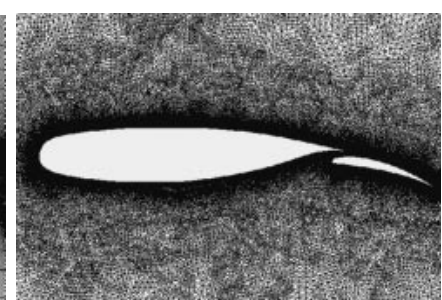

(c) Unstructured mesh of TAS code

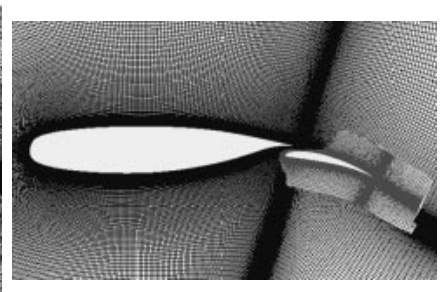

(d) Overset structured mesh of MHI2D

Fig. 2. Meshes used by solvers.

two-equation $k-\varepsilon$ model includes the turbulent transport effect in the model equations, and thus has advantage over algebraic turbulence models for complex flows. However, it is too diffusive, and generally does not perform well for boundary layer flows with separation. The Abid model ${ }^{15)}$ represents a typical low-Re $k-\varepsilon$ model.

In this study, transition is introduced by setting the production terms in the transport equations of turbulence models to zero in the prescribed domain of laminar flow. The transition onset is specified manually according to experimental observation as summarized in Table 2.

\section{Computational Meshes}

In order to minimize the discretization error in the computational results due to the difference of grid refinement between different meshes, all meshes are generated according the following senses learned from the experience of ECARP's research ${ }^{6)}$ for the same test case.

1. The number of grid points on the surface is not less than that of the ECARP's mandatory mesh, where there are 425 points on the surface of the main element and 217 points on the surface of the flap. This number of grids on the surface is generally enough to resolve the variation of the boundary layer in the streamwise.

2. The grid spacing of the first grids adjacent to the surface is set to about $6 \times 10^{-6}$ chord, which corresponds to $\mathrm{y}^{+} \approx 1$ on most of the surface except a small region near the leading edge. This makes the computation correctly resolve the boundary layer including the laminar sublayer, and is capable of integrating directly to the surface.

3. Across the boundary layer, the number of grids is more than 60 within a height of 0.005 chords $\left(y^{+}<\right.$
$500 \sim 800)$ away from the wall. Care is also taken to cluster grid points in the region of the wake downstream to the trailing edges of the main element and flap. This guarantees sufficient resolution within the boundary layer including near wall separation.

4. The outer boundary of the computational domain is extended to more than 100 chords for the uniform freestream boundary condition, and more than 40 chords if a point vortex correction is imposed there. This makes the computational results insensitive to the grid extent for high-lift flows.

Mesh types and sizes for each solver are given in Table 1. The structured mesh shown in Fig. 2(a) is used for UPACS. It consists of 36 blocks. A similar structure mesh (Fig. 2(b)) used for ADCS has 6 blocks. TAS uses an unstructured mesh, as shown in Fig. 2(c). To match the structured grid in accuracy, the number of unstructured grid points used is about twice of that of the structured mesh of ADCS. MHI2D uses an overset structured mesh (Fig. 2(d)) which has 2 blocks around the main element and flap with an overlap between them. Except ADCS, the outer boundaries of the computational domains used for UPACS, TAS and MHI2D are extended to 100 chords away from the airfoil and the uniform free-stream condition is imposed. For ADCS, the grid extent is 40 chords, but a point vortex correction ${ }^{16)}$ is added to the uniform free-stream at the far-field boundary.

\section{Grid Dependency Study}

\subsection{Sensitivity to grid density}

A grid convergence study is a straightforward method to measure the numerical error due to discretization in CFD 


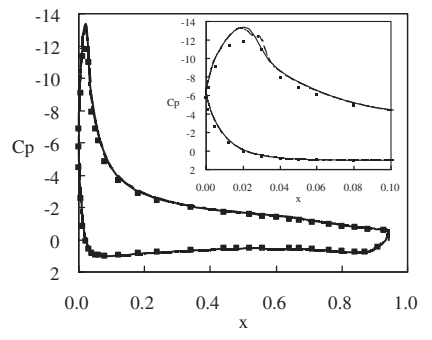

(a) Main element

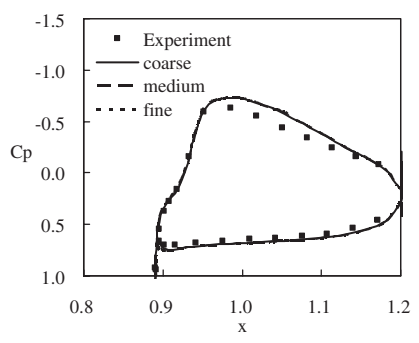

(b) Flap
Fig. 3. Influence of grid density on pressure coefficient on the surface, $\alpha=13.1^{\circ}$.

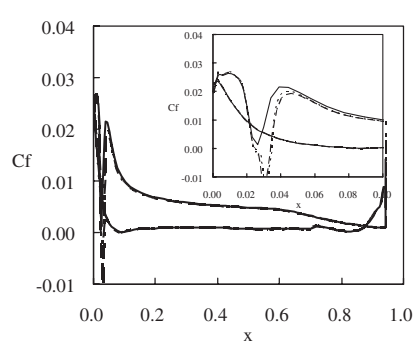

(a) Main element

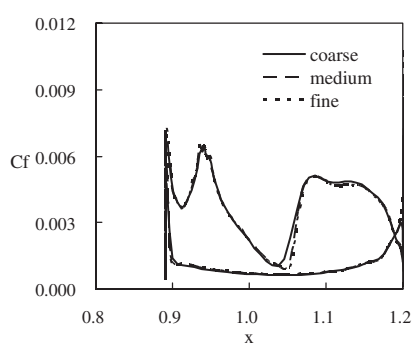

(b) Flap
Fig. 4. Influence of grid density on friction coefficient on the surface, $\alpha=13.1^{\circ}$.

simulation. Discretization error can be reduced by using finer grids in the computation. However, it is expensive to use a large number of grid points. We may want to work on grids with acceptable accuracy as well as good performance. A straight way to analysis the discretization error is to investigate the sensitivity to grid density on computational results. In this study, simulations were conducted on three different levels of structured grids. CFD simulations were performed fully turbulent with UPACS. A fine mesh was generated with very small spacing. The first grid spacing in the normal direction to the airfoil surface is $3 \times 10^{-6}$, which corresponds to $y^{+}<0.5$. This mesh contains about 414,000 grid points in a $2 \mathrm{D}$ plane as given in Table 1 . Then, a medium mesh with 103,000 and a coarse mesh with 26,000 grid points were successively constructed by removing every other grid line in both grid coordinate directions.

Pressure and friction distributions are compared in Figs. 3 and 4, respectively. Due to poor resolution, the coarse grid failed to capture the separation bubble near the leading edge of the main element. Except for the region of the separation bubble, the friction distributions of the three meshes agreed very well with each other. This indicates that the grid spacing of medium and fine meshes in the normal direction to the surface is adequate to calculate the boundary layer near the wall in this computation.

The Richardson extrapolation ${ }^{17)}$ can be used to estimate the grid independence using the results of the three level meshes. The Richardson extrapolation can be generalized for a $p$-th order method and $r$-value of grid refinement ratio as

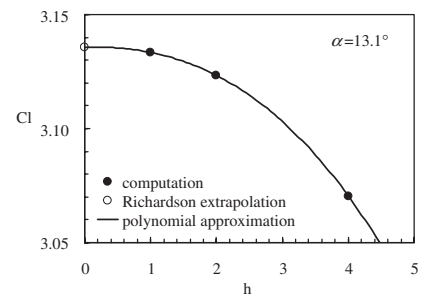

(a) Lift

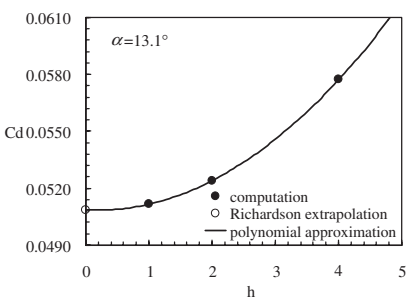

(b) Drag
Fig. 5. Grid convergence of lift and drag, $\alpha=13.1^{\circ}$.

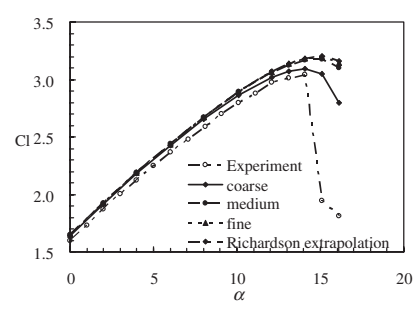

(a) Lift

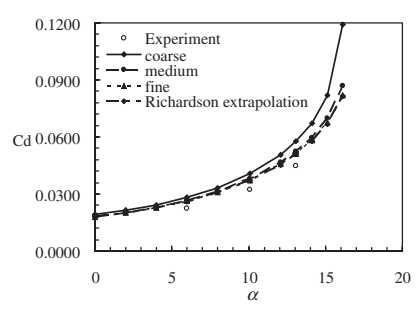

(b) Drag
Fig. 6. Effect of grid density on aerodynamic forces.

$$
f_{h=0}=f_{1}+\frac{f_{1}-f_{2}}{r^{p}-1},
$$

where $p$ can be evaluated from three solutions:

$$
p=\ln \left(\frac{f_{3}-f_{2}}{f_{2}-f_{1}}\right) / \ln (r) .
$$

The values of $f_{1}, f_{2}$ and $f_{3}$ are lift or drag calculated on the fine, medium and coarse meshes, respectively. It provides a 4 th-order estimate of $f_{h=0}$, if $f_{1}, f_{2}$ were computed using exactly the 2nd-order method. In this study, the grid refinement ratio $r$ is exactly equal to 2 . Furthermore, the gridspacing dependent function, say lift or drag, may be fitted by a polynomial approximation. Here, we employ the series expansion at the zero grid spacing of $h=0$.

$$
f=f_{h=0}+g_{1} h+g_{2} h^{2}+g_{3} h^{3}+O\left(h^{4}\right) .
$$

Figure 5 shows an example of the grid convergence calculated at the angle of attack $13.1^{\circ}$. It can be seen that with decreasing the grid spacing, the lift and drag are converged to the values estimated by the Richardson extrapolation, and the tangents at $h=0$ are close to zero. This method was also applied to obtain the grid independent lift and drag at all angles of attack. The effect of grid density on the predicted aerodynamic forces is show in Fig. 6. The coarse mesh presents a large discrepancy from the Richardson extrapolation. Except for the coarse mesh, the trend, variations and even the $C l_{\max }$ at the stall angle of attack calculated on the medium and fine meshes agree very well with the Richardson extrapolation. Further analysis is given for the relative error in Fig. 7. The relative errors of the medium and fine meshes keep less than $2 \%$ in lift and $0.2 \%$ in drag up to the $C l_{\max }$. This indicates that either medium or fine meshes can provide a satisfactory solution without significant discretization error. 


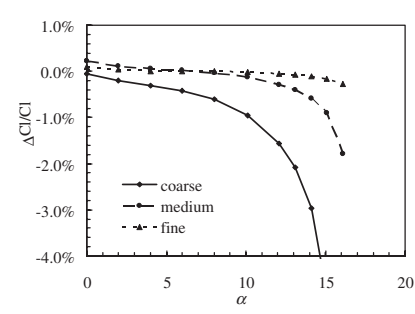

(a) Relative errors of lift

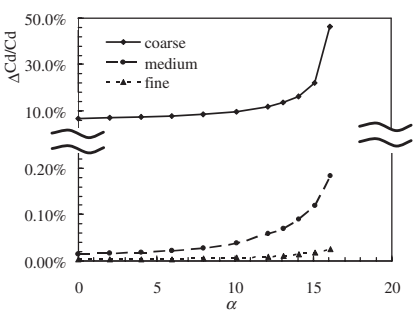

(b) Relative errors of drag
Fig. 7. Error analysis of grid density.

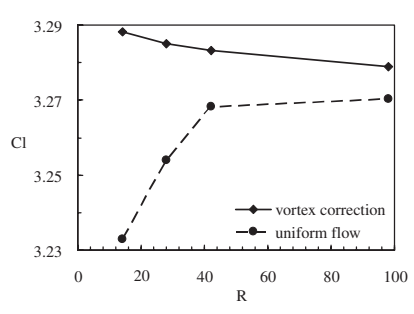

(a) Lift

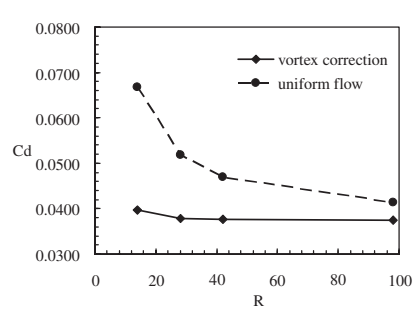

(b) Drag
Fig. 8. Effect of grid extent of the farfield on aerodynamic forces, $\alpha=13.1^{\circ}$

\subsection{Effect of farfield extent and circulation correction}

Dependence of the solution on the size of the computational domain is verified by changing the grid extent of the far-field boundary. Near-field blocks of the meshes are not changed, while away from the airfoil, the grid points are stretched. This ensures that the grid spacing is identical and maintains the same accuracy for all meshes in the near field. CFD simulations were performed for the case $\alpha=$ $13.1^{\circ}$ with fixed transitions using ADCS. At this angle of attack, the lift is near the maximum, and the solution should be more sensitive to the far-field grid extent.

Variations of lift and drag with respect to the far-field grid extent are shown in Fig. 8. By extending the far-field boundary from 14 to 100 chords and applying a uniform flow boundary condition, the computed lift is increased by 0.04 and the computed drag is decreased by 250 drag counts ( 1 drag count $=0.0001$ ). The effect of the grid extent is small on the computed lift, but significant on the computed drag. This shows that the computational domain should be extended more than 100 chords for the uniform flow boundary condition.

An effective remedy for this problem is to include a point vortex model on the far-field boundary condition. ${ }^{16)}$ Velocities at the far-field boundary are perturbed by imposing a potential vortex solution, which is centered at the aerodynamic center of the airfoil, to the free-stream quantities. According to the theory of the point vortex correction, the perturbation to velocity on the far boundary is directly proportional to circulation of the lift and inversely proportional to the distance from the airfoil. Thus, for high lift flow, the larger the lift is, the farther the grid extent should be taken. In Fig. 8, it shows that with the point vortex correction at the far-field boundary, the computed lift and drag converge much more rapidly than the uniform flow condition as the computational domain is extended. The difference between the computed drags of the grid extent 42 and 100 chords is less than 1 drag count. With inclusion of the point vortex correction, the typical grid extent may be about 40 chords away from the airfoil. To accurately predict lift and drag, it is necessary to either have the far-field grid extend far enough or include the point vortex correction in the far-field boundary condition.

\section{Comparison of CFD Solvers}

Any CFD code should be created with correct implementations of numerical method, boundary condition, turbulence model, and so on. A useful method to assess its performance is to compare the accuracy with other solvers. A comparison of RANS computations is conducted using four different codes: UPACS, TAS, MHI2D and ADCS. The Spalart-Allmaras one-equation turbulence model is used to simulate turbulence in all codes.

The computed lift, drag and pitching moment coefficients versus the angle of attack are plotted in Fig. 9(a), (b) and (c), respectively. Differences among computations are very small at most angles of attack. Not only the values, but also the curve slopes predicted by the four solvers are quite close before the stall angle. However, all of the solvers overpredicted the lift. ADCS predicted different values of maximum lift and stall angle most likely because, in ADCS, the production term of the turbulence transport equation is modified with a rotation correction and the computed eddy viscosity may be different from others. The difference in pressure drag is relatively small, as shown in Fig. 10(a). The largest discrepancy in the friction drag (Fig. 10(b)) is found between MHI2D and the others. In MHI2D, the tran-

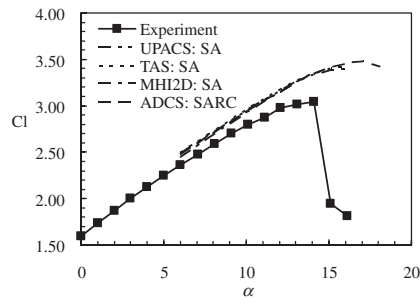

(a) Lift coefficient vs. angle of attack

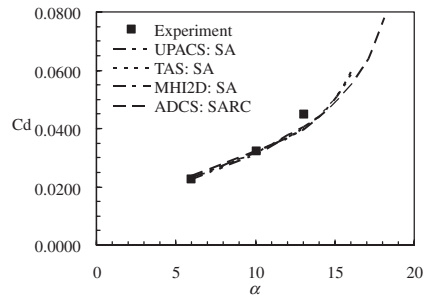

(b) Drag coefficient vs. angle of attack

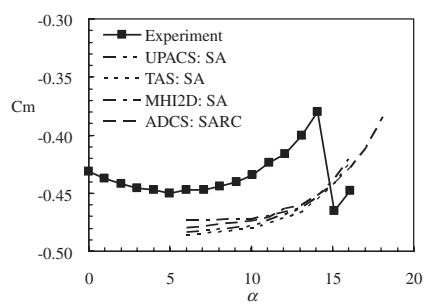

(c) Pitching moment coefficient vs. angle of attack

Fig. 9. Comparison of aerodynamic forces predicted by CFD solvers. 


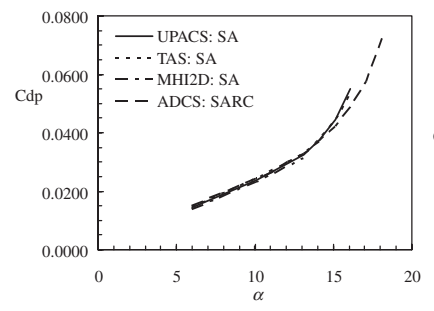

(a) Pressure drag coefficient vs. angle of attack

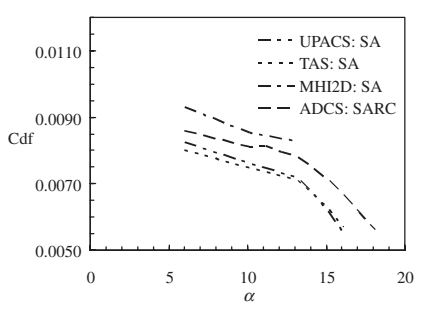

(b) Friction drag coefficient vs. angle of attack
Fig. 10. Comparison of drag components computed by CFD solvers.

sition onset is not specified and full turbulence computation is conducted on the flap. Only a few drag counts are found among friction drags predicted by UPACS, TAS and ADCS.

Distributions of pressure and friction coefficients are compared in Figs. 11, 12 and 13. On most parts of the airfoil, agreement among solvers is quite good. On the other hand, the magnitudes of friction coefficients computed by solvers are different from each other in the regions of the leading edge of the main element and the upper surface of the flap, where large adverse pressure gradients exist. The
Sizes of the laminar separation bubbles were predicted differently. Fortunately, the separation bubble is so small that the prediction accuracy for the separation bubble may have little effect on the aerodynamic forces at small angles of attack. But it is critical for the prediction of the maximum lift.

Eddy viscosities computed by the four solvers are shown in Fig. 14. No significant difference can be seen in these figures. But the eddy viscosity is slightly different within the wakes. Unlike the grids near the airfoil surface, all meshes used in this study are not adapted to the variation in the wake, so the grid spacing is different. This results in some discretization errors. A companion paper ${ }^{2)}$ discusses the effect of the grid refinement within the wake of a three-element airfoil.

\section{Effect of Turbulence Models}

The uncertainty due to turbulence modeling is critical for the use of RANS methods. In order to minimize differences due to numerical discretization errors, we make computations using three turbulence models implemented in the same code, ADCS. Turbulent flows are simulated with the
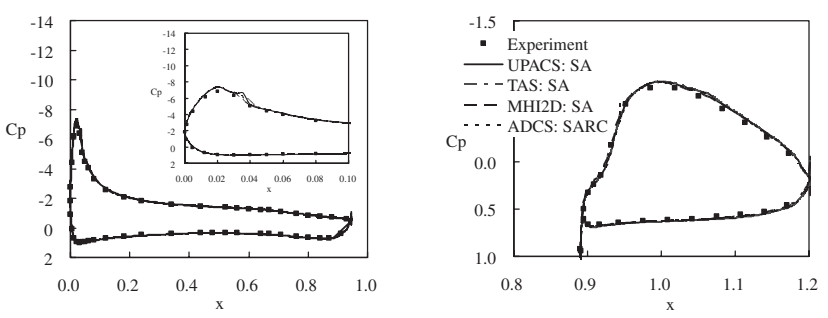

(a) Pressure distributions on the surface
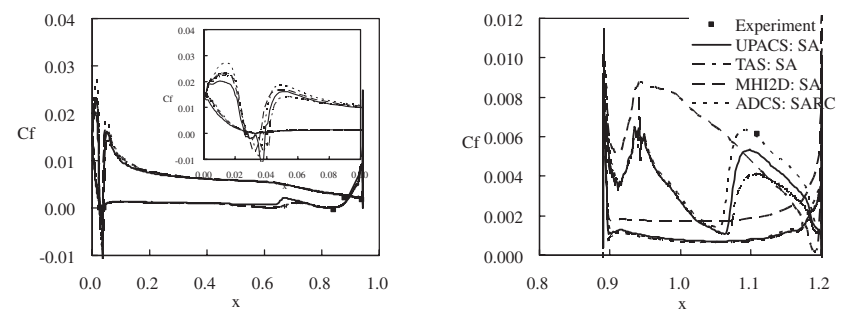

(b) Friction distributions on the surface

Fig. 11. Comparison of solvers, $\alpha=6^{\circ}$. (Left: main element. Right: flap)

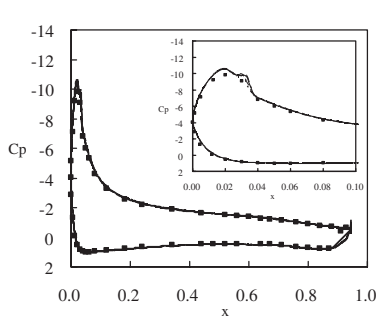

(a) Pressure distributions on the surface
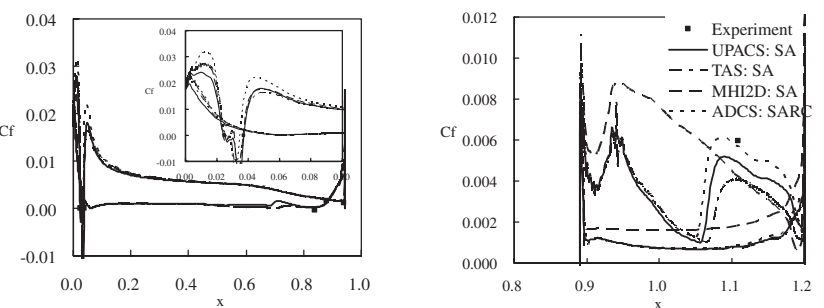

(b) Friction distributions on the surface

Fig. 12. Comparison of solvers, $\alpha=10.1^{\circ}$. (Left: main element. Right: flap)
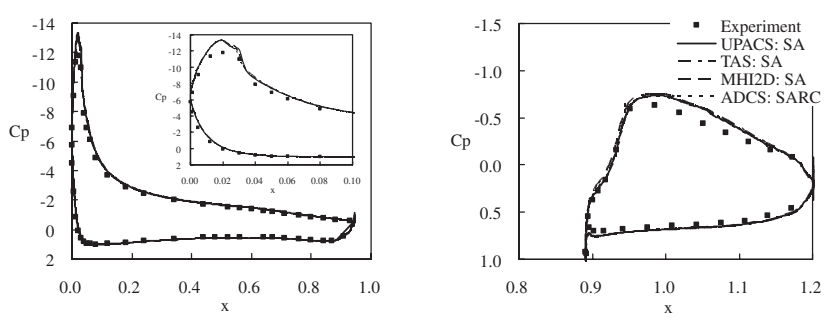

(a) Pressure distributions on the surface
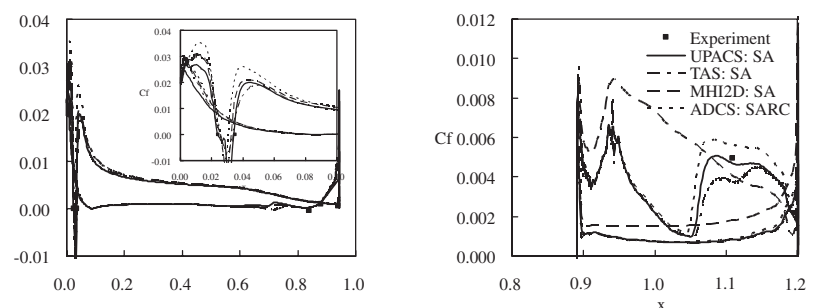

(b) Friction distributions on the surface

Fig. 13. Comparison of solvers, $\alpha=13 \cdot 1^{\circ}$. (Left: main element. Right: flap) 


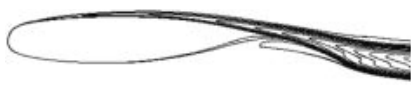

(a) UPACS

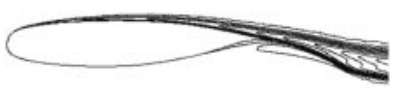

(b) TAS

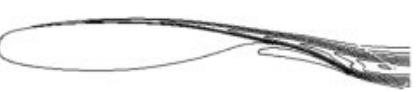

(c) MHI2D

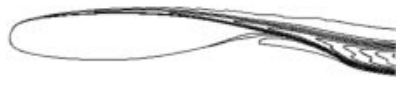

(d) ADCS

Fig. 14. Comparison of eddy viscosities calculated by different codes, $\alpha=13.1^{\circ}$.

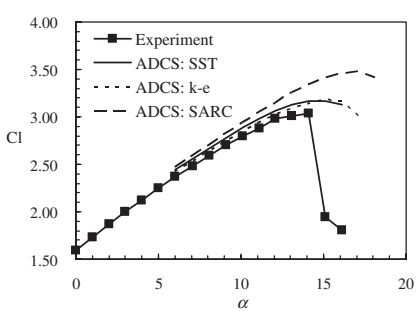

(a) Lift coefficient vs. angle of attack

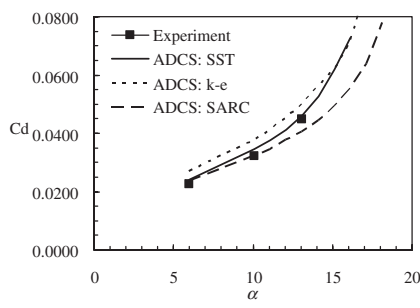

(b) Drag coefficient vs. angle of attack

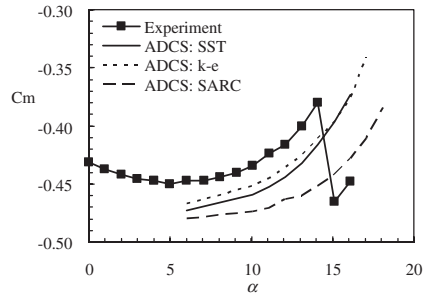

(c) Pitching moment coefficient vs. angle of attack

Fig. 15. Effect of turbulence models on aerodynamic forces.

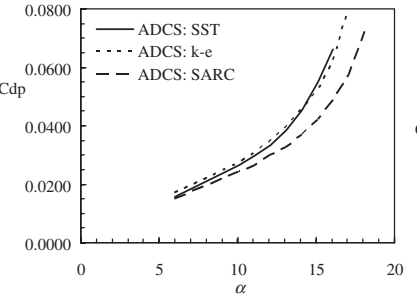

(a) Pressure drag coefficient vs. angle of attack

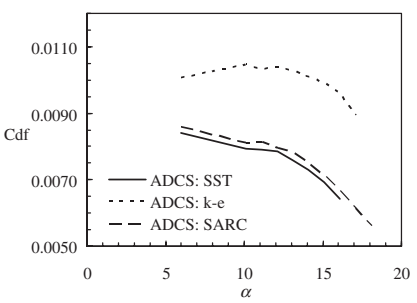

(b) Friction drag coefficient vs. angle of attack
Fig. 16. Effect of turbulence models on drag components.
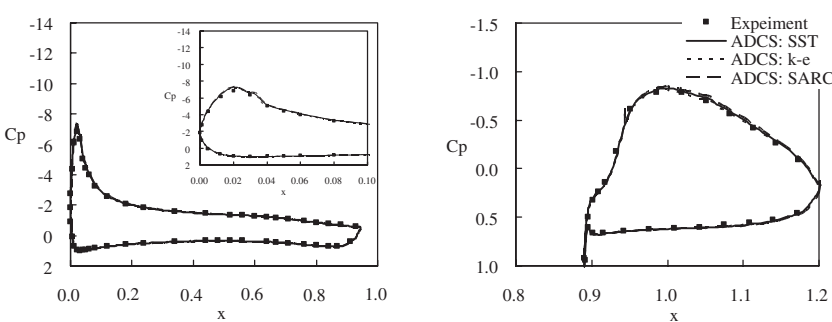

(a) Pressure distributions on the surface
SARC model, Menter's SST $k-\omega$ model and Abid's $k-\varepsilon$ model.

Aerodynamic forces are compared in Fig. 15. All of the turbulence models overpredicted the maximum lift $\mathrm{Cl}_{\max }$ and the stall angle. It is clear that the SST model is in best agreement with the experiment. Both the maximum lift $C l_{\max }$ and the stall angle are close to experiment. Figure 16 shows that the pressure drags predicted by the SST and $k-\varepsilon$ models agree well with each other, but the $k-\varepsilon$ model predicts the friction drag by more than 10 drag counts larger than the SST model does.

Distributions of pressure and friction coefficients are shown in Figs. 17, 18 and 19 at three angles of attack. All three turbulence models computed pressure distributions
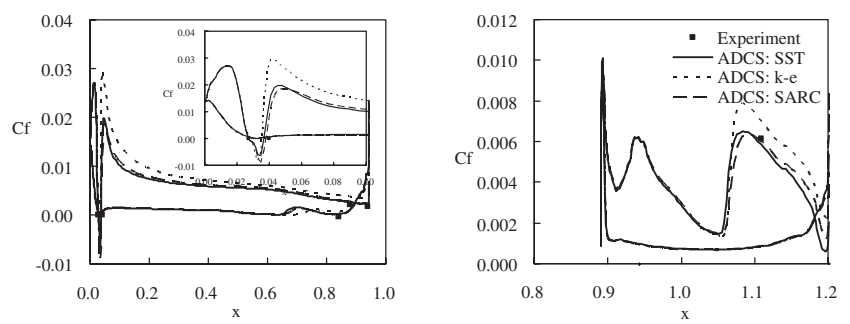

(b) Friction distributions on the surface

Fig. 17. Comparison of turbulence models, $\alpha=6^{\circ}$. (Left: main element. Right: flap)
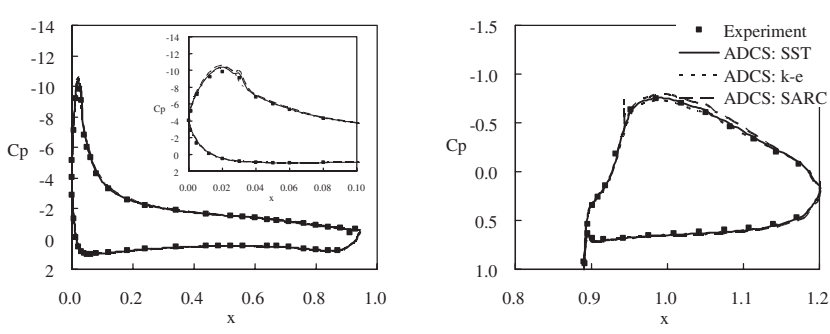

(a) Pressure distributions on the surface
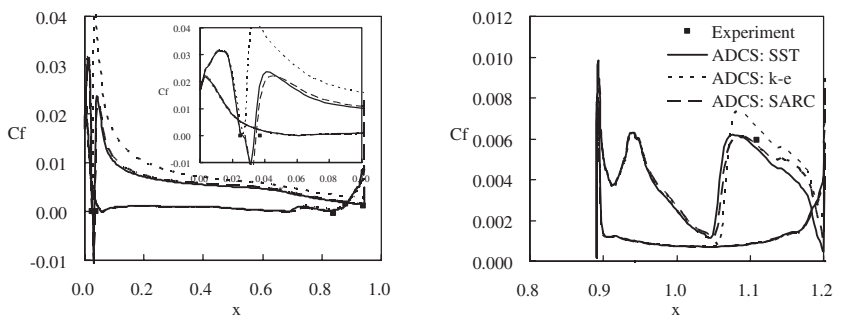

(b) Friction distributions on the surface

Fig. 18. Comparison of turbulence models, $\alpha=10.1^{\circ}$. (Left: main element. Right: flap) 

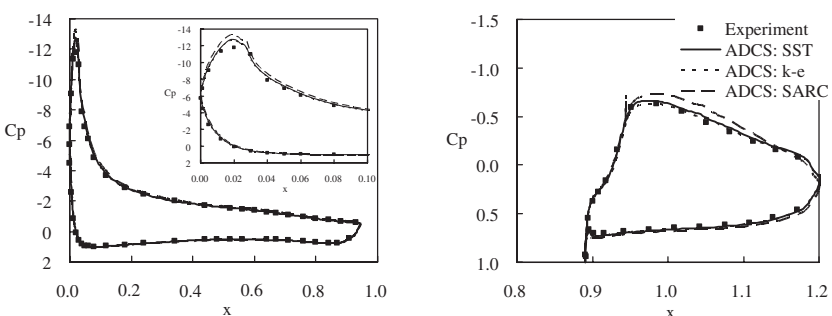

(a) Pressure distributions on the surface
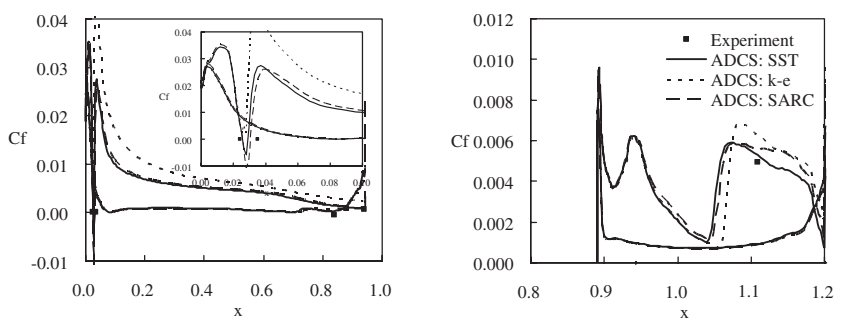

(b) Friction distributions on the surface

Fig. 19. Comparison of results as computed by the same solver using different models, $\alpha=13.1^{\circ}$. (Left: main element. Right: flap)

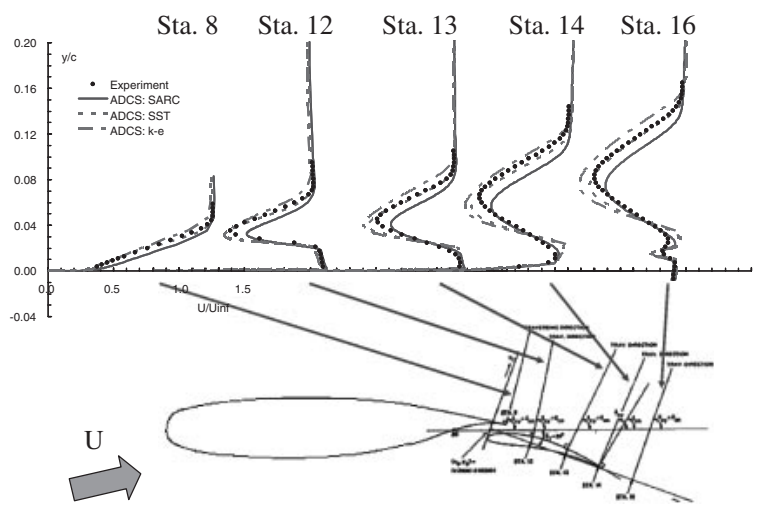

(a) Velocity magnitude

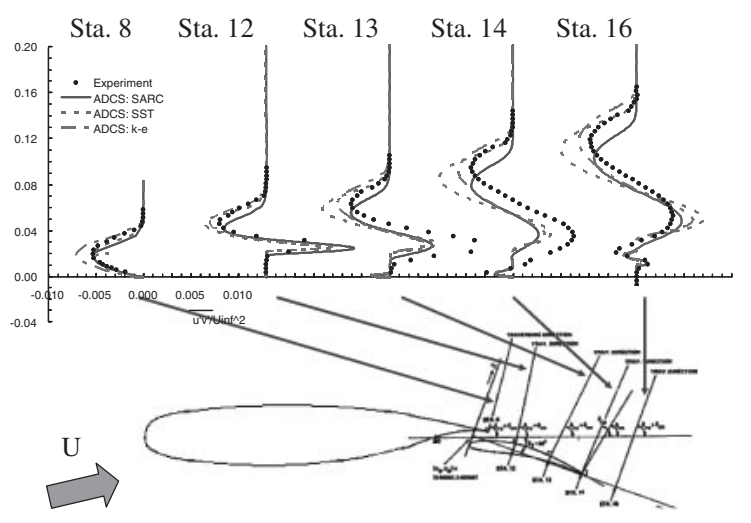

(b) Reynolds shear stress

Fig. 20. Boundary layer profiles, gap $2.6 \%, \alpha=13.1^{\circ}$

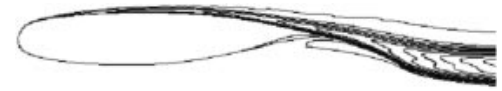

(a) SARC

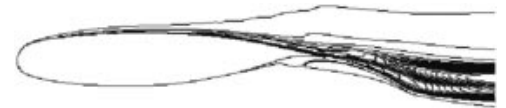

(b) SST

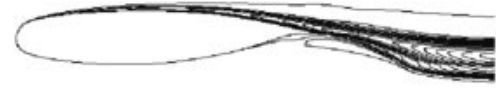

(c) $k-\varepsilon$

Fig. 21. Comparison of eddy-viscosities computed by turbulence models, $\alpha=13.1^{\circ}$.

that agree well with experiment. The level of the suction peaks near the leading edge of the main element is slightly higher than experiment. The SARC model predicts smaller pressure on the suction surface of the flap, thus produces higher lift. The differences between the SARC model and the other two models become clearer as the angle of attack increases. For the skin friction, the SARC and SST models provide satisfactory results. But the $k-\varepsilon$ model predicted significantly high friction. Because the eddy viscosity is predicted too large with the $k-\varepsilon$ model, the laminar separation bubble is rather small at small angles of attack and disappears gradually as the angle of attack increases. Both the SARC and SST models are able to compute the correct separation point of the separation bubble. However, the length of the predicted separation bubbles is shorter than that of experiment because excessive eddy viscosities are computed with the turbulence model.

Boundary layer profiles are compared for a configuration with a gap width of $2.6 \%$ chord at the angle of attack $13.1^{\circ}$. Computed velocity profiles are generally close to the measurements of wind tunnel tests, as shown in Fig. 20(a). Be- cause the wake flow of the main element has the effect of reducing the suction peak on the flap upper surface, it is important to accurately predict the wake flow in the flap design. The general features are well captured. Again, the SST model provides the best result. All turbulence models reproduce the close wake defect and width of the main element near its trailing edge and agree well with experiment well. The computational results become worse further downstream. At the trailing edge of the flap, all three models predict the wake of the main element closer to the flap than experiment. Reynolds shear stresses predicted by the turbulence models are given in Fig. 20(b). All turbulence models are able to capture the features across the boundary layer and wake, but fail to predict the magnitudes of the Reynolds shear stresses. It is difficult to say which turbulence model gives the best result for Reynolds shear stresses.

Finally, the eddy viscosities computed with all three turbulence models are compared in Fig. 21. All turbulence models are agreed well qualitatively, but the magnitudes of the eddy viscosity are different in the wake. 


\section{Conclusions}

CFD validation was conducted for two-dimensional flows around a high-lift two-element airfoil NLR7301. CFD accuracy was discussed. For CFD simulation of high-lift devices, it should take care of grid generation including the grid refinement and grid extent. Sensitivity to grid density is investigated using a series of meshes with different refinements. It shows that satisfactory computational results may be obtained on a medium mesh with acceptable discretization error. The effect of the grid extent of the computational domain is also studied. To accurately predict lift and drag, it is necessary to either have the far-field grid extend far enough, or to include the point vortex correction in the far-field boundary condition. Four different codes with the same turbulence model, the Spalart-Allmaras model, are applied. In spite of different algorithms and mesh types, all four codes predicted quite close results. Differences of predicted pressure and friction distributions on the surface were generally small except separation bubble near the leadingedge. The effects of RANS turbulence modeling were also discussed. It was shown that all turbulence models yielded generally similar results at angles of attack before stall, but they overpredicted the values of the maximum lift as well as the angle at which it occurred. The Menter's SST model predicted the best results of aerodynamic forces, the maximum lift $C l_{\max }$ and the stall angle. The SA model predicted a higher maximum lift $C l_{\max }$, and a larger stall angle. The $k-\varepsilon$ model predicted larger friction drag than the other models.

\section{References}

1) Rumsey, C. L. and Ying, S. X.: Prediction of High Lift: Review of Present CFD Capability, Prog. Aerosp. Sci., 38 (2002), pp. 145-180.
2) Murayama, M., Lei, Z., Mukai, J. and Yamamoto, K.: CFD Validation for High-Lift Devices: Three-Element Airfoil, Trans. Jpn. Soc. Aeronant. Space Sci., 49 (2006), pp. 40-48.

3) Kusunose, K. and Cao, H.: Prediction of Transition Location for a 2-d Navier-Stokes Solver for Multi-Element Airfoil Configurations, AIAA Paper 94-2376, 1994.

4) Krumbein, A.: Automatic Transition Prediction and Application to High Lift Multi-Element Configurations, AIAA Paper 2004-2543, 2004.

5) Van den Berg, B.: Boundary Layer Measurements on a Two-Dimensional Wing with Flap, NLR TR 79009 U, 1979.

6) Haase, W., Chaput, E., Elsholz, E., Leschziner, M. A. and Muller, U. R.: ECARP-European Computational Aerodynamics Research Project: Validation of CFD Codes and Assessment of Turbulence Models, Notes Numer. Fluid Mech., 58 (1997).

7) Takaki, R., Yamamoto, K., Yamane, T., Enomoto, S. and Mukai, J. The Development of the UPACS CFD Environment, High Performance Computing, Proc. of ISHPC 2003, Springer, Tokyo, 2003, pp. 307-319.

8) http://www.ista.jaxa.jp/aet/cfd/cfd-e01.html

9) Lei, Z.: Effect of RANS Turbulence Models on Computation of Vortical Flows over Wing-Body Configuration, Trans. Jpn. Soc. Aeronaut. Space Sci., 48 (2005), pp. 150-160.

10) Nakahashi, K., Togashi, F., Fujita, T. and Ito, Y.: Numerical Simulations on Separation of Scaled Supersonic Experimental Airplane from Rocket Booster at Supersonic Speed, AIAA Paper 2002-2843, 2002.

11) Takenaka, K. and Yamamoto, K.: CFD Analysis and Validation Study of the 2-D High Lift Devices, Proceedings of Aerospace Numerical Simulation Symposium, JAXA SP-04-002, 2004.

12) Spalart, P. R. and Allmaras, S. R.: A One-Equation Turbulence Model for Aerodynamic Flows, AIAA Paper 92-0439, 1992.

13) Spalart, P. R. and Shur, M. L.: On the Sensitization of Turbulence Models to Rotation and Curvature, Aerospace Sci. Technol., 1 (1997), pp. 297-302.

14) Menter, F. R.: Zonal Two Equation $k-\omega$ Turbulence Models for Aerodynamic Flows, AIAA Paper 93-2906, 1993.

15) Abid, R.: Evalutions of Tow-Equation Turbulence Models for Prediction of Transitional Flow, Int. J. Eng. Sci., 31 (1993), pp. 831-840.

16) Thomas, J. L. and Salas, M. D.: Far-Field Boundary Conditions for Transonic Lifting Solutions to the Euler Equations, AIAA J., 24 (1986), pp. 1074-1080.

17) Roache, P. J.: Perspective: A Method for Uniform Reporting of Grid Refinement Studies, ASME J. Fluids Eng., 116 (1994), pp. 405-413. 